contain the increasing trend of Long-term Care Insurance (LTCI) expenditure for the care of aged people with disability. Although the proportion of the budget expenditure for HSDP varies among municipalities, it has been unclear whether these differences are related to the containment of LTCI expenditure. The objective of this study was to examine whether there is some relationship between the proportions of budget expenditure for HSDP and LTCI in all of the municipalities in Japan.

Methods 1640 municipalities were categorised into five groups according to the mean budget expenditure for HSDP per resident aged $\geq 65$ years in $2006-2008(<£ 5, £ 5-<£ 7, £ 7-<£ 9, £ 9-<£ 11$, $\geq £ 11)$. The rate of increase (\%) in LTCI expenditure from 2006 to 2008 was calculated in each category. One-way analysis of variance was used.

Results The rate of increase in LTCI expenditure differed significantly between expenditure categories for $\operatorname{HSDP}(120.7 \%, 120.4 \%$, $119.0 \%, 118.5 \%, 117.0 \%$ from the lowest group $[<£ 5]$ to the highest group $[\geq £ 11]$ respectively; $p<0.001)$. These differences were mainly attributable to significant inverse correlation between budget expenditure for HSDP and the rate of increase in fraction of those who use formal care under LTCI.

Conclusion Municipalities with higher budget expenditure for HSDP have a lower rate of increase in LTCI expenditure, suggesting that HSDP would be a cost-effective service.

\section{P1-357 STATISTICAL ANALYSIS OF SPATIAL SURVIVAL DATA AND ITS APPLICATION TO THE COHORT STUDY OF HIROSHIMA ATOMIC BOMB SURVIVORS}

\author{
doi:10.1136/jech.2011.142976f.49
}

T Tonda, ${ }^{*}$ K Satoh, K Otani, Y Sato, H Maruyama, H Kawakami, S Tashiro, M Hoshi, M Ohtaki. Research Institute for Radiation Biology and Medicine, Hiroshima University, Hiroshima, Japan

Introduction While there are considerable studies on the relationship between the risk of disease or death and direct exposure from the atomic bomb, the risk for indirect exposure caused by residual radioactivity has been ignored. One of the reasons is that risk assessments have utilised estimated radiation dose but it is difficult to estimate indirect exposure. To evaluate risks for indirect radiation exposure as well as direct exposure, we developed a statistical method that evaluates risk with respect to individual location at the time of atomic bomb exposure instead of radiation dose.

Methods Subjects for analysis were selected from the ABS database of the RIRBM at Hiroshima University. The number of death from all causes was 9641 and the number of censoring was 21414 . The follow-up period was from 1980 to 1997. Mesh coordinates of $100 \mathrm{~m}$ in width were used to define location. Sex and age at atomic bomb exposure were used as covariates. Applying the proposed method to the data, we estimated the location-specific risks for direct and indirect exposure.

Result and conclusion The contour of estimated risk showed that the north-west region has higher risk compared to other areas. The resultant risk map, excluding the risk for direct exposure, still has contours skewed towards the north-west direction. These suggest that there exists an impact on risk that cannot be explained by direct exposure. The shape of the risk map, which is skewed towards the north-west direction, might be due in part to Black Rain.

\section{P1-358 RECORD BASED DATA EVALUATION OF 108 -EMERGENCY RESPONSE SERVICES- A HELP TO KNOW THE PATTERN OF EMERGENCIES IN MADHYA PRADESH}

doi:10.1136/jech.2011.142976f.50

N Toppo,* P Kasar, A Bhadoriya. NSCB Medical College, Jabalpur, Madhya Pradesh, India

Introduction GVK EMRI (GVK Emergency Management and Research Institute) is World's Largest Integrated Emergency Response Services (ERS) Provider. It is not-for-profit professional organization operating in the Public Private Partnership (PPP) mode. This is a free service delivered through state- of -art emergency call response centers and has over 2000 ambulances providing necessary professional pre-hospital care while transporting the victim to the nearest hospital. In our state it was launched on 16 July 2009.

\section{Objectives}

1. To determine the type of emergencies reported by 108-ERS.

2. To determine the proportion of emergencies brought to medical college hospital by 108-ERS.

3. To determine the impact of 108-ERS on emergency medical services.

Methods Type of study- Record based data evaluation.

Study area: Four Districts of M.P.

Research period- 1 August 2010 to 31 August 2010.

Tools and techniques: Review of secondary data from 108-ERS, Jabalpur and MRD of NSCB Medical College, Jabalpur.

Results 74557 individuals get benefitted in M.P. till 31 July 2010 from the launch. Out of which 25000 (33.53\%) were pregnancy related emergencies, followed by $16986(22.78 \%)$ of road traffic accidents and 6494 (8.7\%) were of acute abdomen. About one tenth of total emergency cases seen in emergency department of NSCB Medical College, Jabalpur were brought by 108-ERS. Above 6000 lives saved by this 108- Emergency response service in Madhya Pradesh.

Conclusion Record based data are a great help to formulate policies and strategies to improve the health services in any state or country.

\section{P1-359 PUBLIC OPINION OF CHILDCARE PROVIDERS IN ALBERTA, CANADA}

doi:10.1136/jech.2011.142976f.51

\begin{abstract}
${ }^{1,2} \mathrm{~S}$ Tough, ${ }^{*}{ }^{3} \mathrm{~S}$ Rikhy, ${ }^{4} \mathrm{~K}$ Benzies, ${ }^{3,5} \mathrm{M}$ Vekved, ${ }^{3} \mathrm{H}$ Kehler, ${ }^{4,6} \mathrm{D}$ Johnston. ${ }^{1}$ Alberta Centre for Child, Family and Community Research, Calgary, Alberta, Canada; ${ }^{2}$ Departments of Paediatrics \& Community Health Sciences, University of Calgary, Calgary, Alberta, Canada; ${ }^{3}$ Public Health Innovation and Decision Support, Alberta Health Services, Calgary, Alberta, Canada; ${ }^{4}$ Faculty of Nursing, University of Calgary, Calgary, Alberta, Canada; ${ }^{5}$ Department of Paediatrics, University of Calgary, Calgary, Alberta, Canada; ${ }^{6}$ Behavioural Research Unit, Alberta Children's Hospital, Calgary, Alberta, Canada
\end{abstract}

Objective To assess public opinion about, and interactions with, childcare providers and programs.

Methods Between September 2007 and March 2008, 1443 randomly selected adults living in Alberta, Canada, completed a telephone survey. Individuals were eligible to participate if they had interactions with a child $<14$ years of age in the past 6 months.

Results Of the respondents, $52 \%$ believed the government should cover between 40 and $60 \%$ of daycare costs, with $24 \%$ indicating more coverage and 23\% indicating less coverage. Three-quarters $(72 \%)$ indicated that childcare providers at daycare centres should have at least a college diploma. About 80 to $90 \%$ indicated that childcare providers were as central to children's development as elementary school teachers, with females and parents more likely to 